The National Hospital, Center for

Occupational and

Environmental

Medicine, 0027 Oslo,

Norway

S Langård

Norsk Hydro ASA, Hydro Porsgrunn Industripark, 3901 Porsgrunn, Norway $\mathrm{J}$ Rosenberg

Cancer Registry of Norway, Montebello, 0310 Oslo, Norway A Andersen

Vestregate 21, 3900 Porsgrunn, Norway S S Heldaas

Correspondence to: Dr S Langård, The National Hospital, Center for Occupational and Environmental Medicine, 0027 Oslo, Norway

Accepted 13 August 1999

\title{
Incidence of cancer among workers exposed to vinyl chloride in polyvinyl chloride manufacture
}

\author{
S Langård, J Rosenberg, A Andersen, S S Heldaas
}

\begin{abstract}
Based on results from two previous studies where an excess of melanomas was found in a cohort of workers exposed to vinyl chloride (VCM), a follow up of the incidence of cancer in the same cohort of 428 workers was carried out to scrutinise whether or not the excess could be confirmed by new cases. The total number of deaths in the study group from 1953 to the end of 1993 was $132 v 141$ expected, and the total number of incident cancer cases was $56 v 57$ expected. There were 11 cases of lung cancer $v$ eight expected, seven cases of melanomas $v 2.07$ expected, and two cases of thyroid cancer $v 0.34$ expected. Five of the seven melanoma cases had occurred in the group that had been most heavily exposed to VCM $v$ expected. In the present follow up we also found five cases of the spinocellular cancer of the skin $v 1.7$ expected. Out of these five cases four were diagnosed after 1984. Two of the five cases $v 0.7$ expected had occurred in the most heavily exposed group. The total number of skin cancers (melanomas and spinocellular cancers) were $12 v 3.7$ expected. There was one new case of melanoma between 1985 and $1993 v$ 0.7 expected. Hence, the strength of the relation between the observed and expected number of cases was reduced compared with the last follow up, and does not strengthen the previously indicated causal relation between exposure to VCM and development of malignant melanoma. There was no excess of testicular cancers in this study. The present results may indicate that occurrence of spinocellular skin cancer could bear some relation to work in the manufacture of polyvinyl chloride (PVC). Confirmation is needed from studies on other cohorts exposed to VCM.

(Occup Environ Med 2000;57:65-68)
\end{abstract}

Keywords: cohort study; aetiology; exposure; melanomas; spinocellular cancers

Much information is available on the association between exposure of vinyl chloride (VCM) and the occurrence of hemangiosarcomas. $^{1-5}$ The International Agency for
Research on Cancer (IARC) performed a multinational study comprising subcohorts from seven European countries and found excess hemangiosarcomas, and possibly excess cancer of the bladder. ${ }^{6}$ Lung cancer, cancer of the brain, cancer of the thyroid, and melanosarcoma also have been associated with exposure to VCM..$^{3-8}$ Excess incidence of melanomas has been associated with arsenic, polychlorinated biphenyls, alcohol, and polyunsaturated fat. ${ }^{9}$

We have reported twice before on the incidence of cancer from the Norwegian cohort of workers exposed to VCM in the manufacture of polyvinyl chloride (PVC) and VCM. ${ }^{78}$ In the later report ${ }^{8}$ the cohort was followed up to the end of 1984. The incidence of melanoma was found to be in excess in both follow up periods. ${ }^{78}$ To our knowledge there is only one other study that has indicated an excess of melanomas among workers exposed to $\mathrm{VCM}^{10}$; in that study there were five cases versus 1.5 expected.

The aim of the present study was to scrutinise further the incidence of cancer among that same cohort of workers which was followed up in two previous periods, ${ }^{78}$ in particular to study whether an excess of melanomas was still present in an extended follow up period.

\section{Material and methods}

MANUFACTURING PLANT

The PVC plant where the study population was employed is in the county of Telemark in south east Norway. This plant is one of several different chemical manufacturing plants within the same industrial complex, and magnesium, chlorine, and fertilisers are the other main products manufactured during the observation period. Manufacture of VCM and PVC started in 1950. At first VCM was manufactured from acetylene in a small polymerisation reactor. In 1967 the VCM manufacture was altered, cracking of ethylenedichloride (EDC) being introduced as one of two manufacturing methods. The manufacture of VCM was discontinued in 1971 and the plant continued as a polymerisation unit only. Between 1955 and 1971 PVC and VCM were manufactured in separate units. The total volume of VCM manufactured was between 1000 and 2500 tonnes from 1950 to 1955 , between 2500 and 5000 tonnes from 1956 to 1960, and from 
Table 1 Incidence of malignant melanomas, spinocellular skin cancer, and brain tumours in workers exposed to VCM from 1 fanuary 1953 to 31 December 1993

\begin{tabular}{|c|c|c|c|c|c|c|c|c|c|}
\hline \multirow[b]{2}{*}{ Tumour site } & \multicolumn{2}{|c|}{$<1$ ppm-years } & \multicolumn{2}{|c|}{ 1-5 ppm-years } & \multicolumn{2}{|c|}{$\geqslant 5$ ppm-years } & \multicolumn{2}{|c|}{ Total } & \multirow[b]{2}{*}{$95 \% C I$} \\
\hline & Obs & $\operatorname{Exp}$ & Obs & $\operatorname{Exp}$ & Obs & Exp & Obs & $\operatorname{Exp}$ & \\
\hline Lung cancer (162) & 5 & 3.0 & 1 & 1.46 & 5 & 3.50 & 11 & 7.99 & 0.65 to 2.46 \\
\hline Malignant melanoma (190) & 2 & 0.8 & 0 & 0.54 & 5 & 0.73 & 7 & 2.06 & 1.36 to 6.96 \\
\hline Spinocellular cancer (191) & 2 & 0.6 & 0 & 0.32 & 3 & 0.74 & 5 & 1.67 & 0.97 to 6.98 \\
\hline Brain tumours (193) & 1 & 0.7 & 0 & 0.40 & 1 & 0.74 & 2 & 1.78 & 0.14 to 4.05 \\
\hline
\end{tabular}

5000 to 30000 tonnes between 1961 and 1970. The corresponding tonnage of PVC was about the same as for VCM up to 1970 and from 1971 and onwards increased from 30000 to 60000 tonnes. Both suspension and emulsion PVC were manufactured.

\section{STUDY POPULATION}

The methods for compiling the study cohort have been reported previously. ${ }^{78}$ In brief, a list of all employees engaged in the manufacture of VCM and PVC was compiled from personnel records and from medical records that were retrievable in the plant's health department from 1940 onwards. By combining these two sources of information it was possible to identify all consecutive workplaces continuously for each individual employee. The following individual information was compiled: full name; date of birth; the unique identification number from 1964 onwards; the last known address; dates of start and end of employment in the different departments of the plant complex; and the duration of work in each department. All workers included were identified at the end of the 1970s. These data were compiled for 1233 workers first employed between 1950 and the end of 1974 . In the analysis phase, workers included were restricted to the following criteria: employment exceeding 12 months, and first employment at the plant before 1970. This reduced the number of workers to $430 .{ }^{8}$ Another two subjects seemed to be duplicates, reducing the restricted number to 428 subjects in the present follow up.

ESTIMATES OF EXPOSURE

The estimation of exposure to VCM was reported earlier. ${ }^{78}$ As no industrial hygiene surveys had been carried out before 1974 the exposure had to be estimated. Information on exposure was derived from the following sources: (a) measurements resulting from the use of an explosion meter with a scale from $0 \%$ to $100 \%$, which was used in the 1950 s and 1960 s to estimate the lower explosion limit at $4 \%$ (40000 ppm) of VCM; (b) a suggested odour threshold at about $500 \mathrm{ppm}$ combined with interviews with previous workers; and (c) interviews with workers in 1979-80, some of whom had been employed in the plant since the start of production. Based on this information it was estimated that the VCM concentration varied around $2000 \mathrm{ppm}$ between 1950 and $1954 ; 1000 \mathrm{ppm}$ in the subsequent 5 years; around $500 \mathrm{ppm}$ from 1960 to 1967; and from 1968 to 1974 the concentration in the autoclave area was estimated to be about 100 ppm. During cleaning of autoclaves other authors $^{11}$ suggested concentrations of $\geqslant 3000$ ppm. Before 1970 it is likely that a surplus of monomer was present in the PVC when leaving the manufacturing plant; which could have been $\geqslant 500 \mathrm{ppm}$ VCM. For some weeks after polymerisation some remaining monomer could still be released from PVC. The plant closed for reconstruction in October 1974 and was reopened in February 1975. From 1975 the concentrations of VCM in the manufacturing units has been monitored continuously and has generally been $<1 \mathrm{ppm}$, with some peaks up to 5-10 ppm.

\section{JOB CLASSIFICATION}

As in the previous follow ups, ${ }^{78}$ nine different job categories related to exposure were defined; (1) vinyl chloride (acetylene); (2) vinyl chloride (EDC); (3) research laboratory; (4) polyvinyl chloride manufacture; (5) autoclave cleaning; (6) maintenance; (7) drying and packing; (8) customers service laboratory; and (9) various jobs. Some workers had served in more than one exposure category; therefore, assignment to category was defined as the category in which a subject had served the longest period.

\section{EXPOSURE CLASSIFICATION}

We applied the same job classification in the present follow up as was used in the two previous follow ups, where the background for the classification was presented. ${ }^{78}$ The job categories are shown in table 1 , which also includes the crude results. An additional classification of exposure ppm-years was applied ${ }^{7}$ to account for the difference between the high concentrations of exposure during the 1950s, the reduced concentrations during the 1960s, and the very low concentrations from 1975 onwards. For each year a subject was exposed to concentrations suggested to be $2000 \mathrm{ppm}$ in the early 1950s. Thus, each year of exposure counts for 4 years of exposure to $500 \mathrm{ppm}$. Conversely, exposure after 1968 (at about 100 ppm) counts only $1 / 5$ year of $500 \mathrm{ppm}$.

EPIDEMIOLOGICAL METHODS

All workers who met the inclusion criteria were included in the present follow up. The Cancer Registry of Norway has recorded all new cases of cancer in the total population from 1953 and onwards, and its register of new cancers is considered to be $>99 \%$ complete. Dates of death and dates of emigration were available from the information compiled at The National Central Bureau of Statistics. The identification of cancer cases is based on a linkage between the register of members of the cohort and the data on incident cancers in the register of cancers in the Registry; any new case of cancer that occurred among the cohort members between 1 January 
1953 and the end of 1993 is registered at the Registry and could thus be identified through the linkage. The age related incidence data at the Registry were also used as a source of reference to calculate expected incidence of cancers in the study population.

\section{STATISTICS}

It was assumed that the cancer cases had occurred in a Poisson distribution, and confidence intervals were derived from this assumption.

\section{Results}

The present follow up included 428 workers first employed between 1950 and the end of 1969 , all participating for $\geqslant 1$ year in the manufacture of VCM and PVC. All workers who met the inclusion criteria were identified at the end of the 1970s. The first two follow up periods covered the periods 1953-79 and 1953-84, whereas the present covers the period from 1 January 1953 to the end of 1993.

The total number of deaths in the study group was 132 versus 141 expected, and the total number of incident cases of cancer was 56 versus 57 expected. There were 11 cases of lung cancer versus eight expected, seven cases of melanomas versus 2.07 expected, and two cases of thyroid cancer versus 0.34 expected. Five versus 0.7 expected (95\% confidence interval (95\% CI) 2.3 to 16.7$)$ out of the seven cases of melanomas had occurred in the group that had been most heavily exposed to VCM (table 1). The corresponding figures in the previous study ${ }^{8}$ were four and six, respectively. In this follow up five cases of spinocellular cancer of the skin were observed versus 1.7 expected (table 1). Three (one expected) of these five cases were diagnosed after 1 January 1984, and of those, two cases versus 0.7 expected had occurred in the most heavily exposed group (table 1). The total number of skin cancers, melanomas and spinocellular cancers combined, were 12 versus 3.7 expected (95\% CI 1.7 to 5.7 ).

Only one new case of melanoma was diagnosed between 1985 and 1993, versus 0.7 expected. Hence, the ratio between observed and expected number of cases was slightly reduced since the last observation. ${ }^{8}$ However, it seems worth mentioning that one additional case of melanoma of the skin was diagnosed outside the observation period in one subject in the autumn of 1996 . The case subject was born in 1919 and worked at the PVC research laboratory from 1953 to the end of 1974 and belonged to exposure category $03,{ }^{7}$ the intermediate exposure concentration 1-5 ppmyears, where the expected figure (table 1) up to the end of 1993 was 0.54 case.

There were no new cases of hemangiosarcoma of the liver as well as the one case that had occurred in the cohort in the early 1970s, neither had any new case of cancer of the thyroid occurred during the observation period 1985-93. Hemangiosarcoma of the liver was considered to be a signal cancer for exposure to VCM from the early 1970 s onwards. ${ }^{23}$
The locations of the melanomas diagnosed previously have been presented before ${ }^{7}$; three on the trunk, two in the face-neck area, and on a foot. The new case of melanoma was located on the upper back, and the case not included in the study was located in the lumbar region. The total number of person-years was 12281 , of which 4559 were in the group with $<1 \mathrm{ppm}-$ years, 3380 in the group with 1-5 ppm-years, and 4343 in the group with $>5$ ppm-years.

\section{Discussion}

In the two previous follow up studies ${ }^{78}$ an excess incidence of melanomas was observed. The present study confirms the previous finding in that one more case was found in one of the case subjects who had contracted melanoma in the previous studies (table 1)..$^{8}$ There is only one other cohort study ${ }^{9}$ that has found an excess of incidence of melanoma among workers exposed to VCM. When accounting for the new case diagnosed in 1996 (outside the observation period) two cases had occurred versus about 1.3 cases expected. All but one of the seven melanoma cases in the observation period occurred after quite a short latent period of 17.4 years (range 2-33 years). The time of diagnoses for these cases could be given because all case subjects were followed up by the occupational health services at the plant. Given that the latent period is short for melanomas, therefore, a causal relation between exposure to VCM and development of this tumour does not conflict with the present results, which seem to indicate higher incidence in the early than in the late phase of the observation period. There is no clear pattern in the scientific literature on the duration of the latent period for malignant melanoma when associated with other exposure factors. However, if the results in the extended observation period are considered separately, the present melanoma results would not strengthen the assumption of causality between exposure to VCM and the occurrence of melanomas. The lack of excess incidence of melanoma in the extended observation period (1985-93) might be considered to be in line with a view that level of exposure to VCM after October 1974 has been too low to result in new cases. However, the additional case occurring in 1996 may be considered to disturb that indication.

It may be worth noting that the reports that have indicated an excess incidence of melanomas $^{7810}$ all were based on incidence based tumour registers, and not on mortality data only. As the survival rate after surgery for melanoma varies considerably from one country to another, it is conceivable that a possible causal relation between exposure to VCM or other chemicals and melanoma or other skin tumours, could be more readily shown in countries with comprehensive incidence based cancer registers, compared with countries with mortality data only. Therefore, the present results need confirmation by studies in other cohorts exposed to VCM, preferably incidence based cancer studies.

Superficially the reduced incidence of melanoma during the 9 years of follow up from 
Table 2 Incidence of cancer at different sites in workers exposed to VCM; observation period 1953-93

\begin{tabular}{|c|c|c|c|c|c|c|c|c|c|}
\hline \multirow[b]{2}{*}{ Tumour site } & \multicolumn{2}{|c|}{$<1$ ppm-years } & \multicolumn{2}{|c|}{ 1-5 ppm-years } & \multicolumn{2}{|c|}{$\geqslant 5$ ppm-years } & \multicolumn{2}{|c|}{ Total } & \multirow[b]{2}{*}{$95 \% C I$} \\
\hline & Obs & $\operatorname{Exp}$ & Obs & $\operatorname{Exp}$ & Obs & $\operatorname{Exp}$ & Obs & $\operatorname{Exp}$ & \\
\hline Stomach cancer (151) & 2 & 1.6 & 2 & 0.77 & 1 & 2.05 & 5 & 4.41 & 0.37 to 2.65 \\
\hline Colon cancer (153) & 2 & 1.7 & 2 & 0.88 & 2 & 2.0 & 6 & 4.63 & 0.48 to 2.82 \\
\hline Prostate cancer (177) & 3 & 3.4 & 0 & 1.67 & 3 & 4.48 & 6 & 9.68 & 0.23 to 1.35 \\
\hline Thyroid cancer (194) & 0 & 0.13 & 0 & 0.08 & 2 & 0.13 & 2 & 0.34 & 0.71 to 21.34 \\
\hline Unspecified cancer (178) & 0 & 0.8 & 1 & 0.4 & 1 & 0.99 & 1 & 2.19 & 0.02 to 2.54 \\
\hline Testes $(178)$ & 0 & 0.29 & 0 & 0.25 & 1 & 0.21 & 1 & 0.75 & 0.03 to 7.43 \\
\hline
\end{tabular}

1985 onwards, compared with the previous observation periods, could have been brought about by the reduction in exposure to VCM to $<2$ ppm from 1974 onwards. However, it is possible to make that inference only if it there is a causal relation between exposure to (high concentrations of) VCM and the occurrence of melanomas. Such causality is not confirmed in a large international study. ${ }^{6}$ On the other hand, the overall excess of skin cancers, as observed in the present results, may indicate some type of relation between exposure to VCM and cancer of the skin. Based on the present results, nothing can be said about possible mechanisms of such a relation. Furthermore, the excesses of both types of cancers could still be due to chance.

The results from the present follow up also indicate that occurrence of spinocellular skin cancer could be related to work in manufacture of PVC. Constituents of PVC cannot be excluded as causal factors. The mean development time for spinocellular skin cancer cannot be given specifically because regulations in the permission from the Data Inspectorate prevents that. However, as exposure for all case subjects took place between early 1950 and 1969 , and all but one case occurred around 1990, the development time must be between 25 and 30 years.

In the present study only one case of testicular cancer was found (table 2). Such a result does not give support to a hypotheses that cancer of the testes is related to exposure factors encountered in manufacture of products containing PVC, as hypothesised in a recent casereferent study. ${ }^{13}$

We thank Liv O Rørvik for invaluable technical assistance in the compilation of the data. The study has been supported by the Department of Occupational Health, Norsk Hydro ASA.

1 Creech JL, Johnsen MN. Angiosarcoma of liver in the manufacture of polyvinyl chloride. f Occup Med 1974;16:150-1.

2 Tabershaw IR, Gaffey WR. Mortality study of workers in the manufacture of vinyl chloride and its polymers. $f$ Occup Med 1974;16:509-18.

3 Spirtas R, Kaminiski R. Angiosarcoma of the liver in vinyl chloride/polyvinyl chloride workers. 1977 update of the NIOSH register. F Occup Med 1978;20:427-9.

4 Waxweiler RJ, Stringer W, Wagoner JK, et al. Neoplastic risk among workers exposed to vinyl chloride. Ann NY Acad Sci 1976;271:40-8.

5 Fox AJ, Collier PF. Mortality experience of workers exposed to vinyl chloride monomer in the manufacture of polyvinyl chloride in Great Britain. Br F Ind Med 1977;34:1-10.

6 Simonato L, L'Abbe KA, Andersen Aa, et al. A collaborative study of cancer incidence and mortality of vinyl chloride workers. Scand f Work Environ Health 1991;17:159-69.

7 Storetvedt Heldaas S, Langård S, Andersen Aa. Incidence of cancer among vinyl chloride and polyvinyl chloride workers. Br f Ind Med 1984;41:25-30.

8 Storetvedt Heldaas S, Andersen Aa, Langård S. Incidence of cancer among vinyl chloride and polyvinyl chloride workers; further evidence for an association with malignant melanoma. Br f Ind Med 1987;44:278-80.

9 Sober AJ, Fitzpatrick TB. Genetic and environmental factors of malignant melanoma in man. Pigment Cell Res 1979;5:88-94.

10 Lundberg I, Gustavsson A, Holmberg B, et al. Mortality and cancer incidence among PVC-processing workers in Sweden. Am F Ind Med 1993;23:313-9.

11 Barnes AW. Vinyl chloride and the production of PVC. Proceedings of the Royal Society of Medicine 1976;69:277-81.

12 Karstadt M. PVC: health implications and production trends. Environ Health Perspect 1976;17:107-15.

13 Hardell L, Ohlson C-J, Fredrikson M. Occupational exposure to polyvinyl chloride as a risk factor for testicular exposure to polyvinyl chloride as a risk factor for testicular
cancer evaluated in case-control study. Int 7 Cancer 1997;73:828-30. 